\title{
Isoniazid toxicity in a 5-year-old boy
}

\author{
Michelle Science MD, Shinya Ito MD, Ian Kitai MD
}

Competing interests: Shinya Ito is the recipient of grant funding from the Canadian Institutes of Health Research for researching the detection of genetic markers for adverse drug reactions. No other competing interests were declared.

This article has been peer reviewed.

\section{Correspondence to:} Ian Kitai, ian.kitai@sickkids.ca

CMAJ 2013. DOI:10.1503 /cmaj.121732

1 ing medical conditions. 5-year-old boy was seen in a tuberculosis clinic 1 week after his mother had received a diagnosis of fully sensitive active pulmonary tuberculosis. The patient's past medical history was unremarkable, with no previous admissions to hospital and no underly-

The results of the patient's initial evaluation included a normal history, physical examination, and radiograph of the chest, as well as a negative result on a tuberculin skin test. At a subsequent evaluation 8 weeks later, the patient's tuberculin skin test had a positive result $(6 \times 9 \mathrm{~mm})$. A repeat chest radiograph was normal. We diagnosed latent tuberculosis infection.

After we discussed the benefits and risks of treatment, we prescribed isoniazid $(11 \mathrm{mg} / \mathrm{kg}$ daily $[200 \mathrm{mg} / \mathrm{d}])$ and vitamin $B_{6}(12.5 \mathrm{mg} / \mathrm{d})$. Test results showed normal baseline levels of liver enzymes. We followed the patient according to current recommendations. ${ }^{1}$ At each monthly visit, he underwent a clinical assessment and physical examination, and we reviewed adverse effects and discussed the warning signs of medication toxicity with the patient's family.

At his 5-month visit, the patient was noted to have upper respiratory symptoms but was otherwise well. Two weeks later, vomiting and abdominal pain developed. The patient's family had believed his complaints were related to school aversion and had continued to administer isoniazid for at least 10 days after the symptoms began. The patient's mother had just completed treatment (daily directly observed therapy) with a public health nurse. When her son's symptoms persisted, she contacted the public health nurse,

\section{KEY POINTS}

- The most common complications of isoniazid toxicity involve the liver.

- Hepatotoxicity can occur at any point during the course of treatment.

- Isoniazid should be withheld immediately at the onset of anorexia, jaundice, vomiting or abdominal pain.

- Information on important adverse effects and a clear plan of action if they occur should be communicated to patients verbally, via a translator if necessary, and in writing. who advised withholding isoniazid immediately and made an urgent referral to the tuberculosis clinic.

At the tuberculosis clinic, the patient had visible jaundice but appeared otherwise healthy. Laboratory investigations showed high levels of aspartate transaminase (AST; 3576 [normal < 45] U/L), alanine transaminase (ALT; 2546 [normal $<40$ ] U/L) and conjugated bilirubin (147 [normal 0-2] $\mu \mathrm{mol} / \mathrm{L}$ ), and an international normalized ratio (INR) of 1.7. The patient was urgently referred to the gastroenterology service and admitted to hospital for evaluation, monitoring and treatment. Ultrasonography of his liver showed increased periportal echoes and coarse echogenicity consistent with acute hepatitis. A transjugular biopsy of the patient's liver showed subacute necrosis with micronodular cirrhosis. Toxicology screening had negative results, and acetaminophen was not detected in the patient's blood. Serologic tests for hepatitis A, B and C viruses, HIV, Epstein-Barr virus and cytomegalovirus were all negative, as was polymerase chain reaction testing for Epstein-Barr virus, cytomegalovirus, herpes simplex virus, varicella zoster virus and parvovirus. Thus, the most likely diagnosis was drug-induced hepatitis. The patient's family said that he had not taken other prescription, over-the-counter or herbal medications. The possibilities of pharmacy errors and inadvertent overdosing were investigated and ruled out.

During the patient's stay in hospital, he received supportive treatment. His AST level peaked at $4066 \mathrm{U} / \mathrm{L}, 3$ days after isoniazid had been discontinued; his ALT level peaked at 2895 U/L, 5 days after isoniazid had been discontinued. The patient was placed on a waiting list for liver transplantation when his INR peaked at 2.3 (9 d after discontinuing isoniazid) and signs of hepatic encephalopathy developed. However, the patient's INR spontaneously improved, as did his clinical condition, and he was removed from the transplant list 13 days after discontinuing isoniazid. On discharge from hospital 3 weeks after admission, the patient's liver function was improving (ALT $414 \mathrm{U} / \mathrm{L}$, AST $570 \mathrm{U} / \mathrm{L}$ and INR 
1.7). His INR returned to a normal value 1 month after he stopped taking isoniazid, and his transaminase levels returned to normal 6 months later.

\section{Discussion}

Our patient presented with hepatic failure during the fifth month of isoniazid treatment for latent tuberculosis infection. For much of the duration of his treatment, the patient showed no symptoms of toxicity, and he continued receiving the medication for at least 10 days after the onset of symptoms. Although the level of isoniazid in his blood was not measured, he was receiving an appropriate dose (recommended range 10-15 $\mathrm{mg} / \mathrm{kg}$ daily [maximum dose $300 \mathrm{mg} / \mathrm{d}$ ]). Furthermore, reactions appear to be idiosyncratic, occurring independent of dose. ${ }^{2}$

Isoniazid is a synthetic antibiotic that inhibits mycolic acid synthesis, a key component of the mycobacterial cell wall. The drug is mainly metabolized in the liver by $\mathrm{N}$-acetyltransferase 2 and cytochrome P450 2E1 and is the only agent established by randomized trials as effective for the treatment of latent tuberculosis infection in children. ${ }^{3}$ American and Canadian guidelines advocate targeted testing for latent infection of close contacts of patients with tuberculosis, ${ }^{3,4}$ as well as new immigrants from countries where the disease has a high prevalence, and people with a positive result on a tuberculin skin test should receive treatment with isoniazid. ${ }^{3}$

Adverse events associated with isoniazid may affect many organ systems. Among the most common adverse effects are those affecting the liver with varying severity: a mild elevation in transaminase levels may occur in $15 \%-20 \%$ of patients $;{ }^{5}$ clinical hepatitis or hepatotoxicity $(0.5 \%-2.0 \%)$ is more concerning. ${ }^{6}$ Fatal hepatitis is rare (about $0.001 \%$ of patients), ${ }^{7}$ but both liver transplantation and death have occurred in adult and pediatric populations.

\section{Risk factors for liver toxicity}

Several risk factors for liver toxicity have been identified, including HIV infection, daily alcohol consumption, age over 34 years, pregnancy and the postpartum period (within 3 mo of delivery), concomitant use of other hepatotoxic drugs and underlying liver disease. ${ }^{4,8}$ The incidence of toxicity increases with age, ${ }^{8}$ although age has not been found to affect the incidence of isoniazid hepatotoxicity in studies involving pediatric patients. 9 Slow acetylation of isoniazid due to genetic polymorphisms in the gene encoding $N$ acetyltransferase 2 and polymorphisms of cytochrome P450 2E1 that result in increased activity of the enzyme have both been associated with raised levels of transaminases during therapy. Unfortunately, there is no validated genotype testing for these variants, and their contribution to the burden of isoniazid hepatotoxicity remains controversial.

\section{Monitoring}

In the absence of sensitive and specific predictors of hepatotoxicity, the optimal monitoring strategy for patients taking this drug is controversial. Current guidelines in both the United States ${ }^{8}$ and $\mathrm{Canada}^{4}$ recommend that clincal monitoring be done at routine follow-up visits and that laboratory monitoring at baseline and monthly thereafter be reserved for patients at high risk of hepatitis.

Our patient's case illustrates several key points with respect to monitoring. First, even when protocol is followed, severe isoniazidassociated liver injury can still occur and may not be detected early enough. Our patient returned for follow-up monthly according to the established protocol, but his condition was not identified before severe liver injury had developed. Similar results were documented in a retrospective review of cases of isoniazid-associated liver toxicity by the Centers for Disease Control and Prevention (CDC), where 17 severe adverse events including 5 deaths and 5 liver transplants were documented over 4 years among patients who were followed according to the American Thoracic Society and CDC guidelines. ${ }^{3,8}$ These patients included people with and without a putative predictor for isoniazid-associated liver injury. Continued use of isoniazid despite symptoms was a common factor in this case series.

Second, hepatotoxicity can occur at any time after the start of treatment and may not be preceded by a gradual increase in liver enzyme levels. Severe hepatotoxicity developed in the fifth month of our patient's therapy despite normal liver enzyme levels at baseline. Unlike other hypersensitivity reactions, hepatotoxicity often occurs weeks to months after isoniazid is started ${ }^{8}$ and should be considered at any point during the course of treatment if symptoms develop. Patients and caregivers should be educated to maintain vigilance throughout the course of treatment, as suggested by the American Thoracic Society Guidelines. ${ }^{8}$

Third, we have found that children can appear healthy despite impending serious or fatal hepatic toxicity. The degree of elevation in liver enzyme levels may not correlate with symptoms. Nausea, vomiting and abdominal pain are common, nonspecific symptoms that might not trigger much initial concern for parents or patients without strong guidance. 
Finally, our patient's isoniazid treatment was continued for at least 10 days after the onset of symptoms suggestive of liver disease. Although discontinuation of the medication at the onset of symptoms does not ensure recovery, ${ }^{10}$ continued use of isoniazid after the onset of symptoms has been associated with poor outcome and death. ${ }^{11}$ Therefore, current recommendations are that isoniazid should be withheld as soon as any symptoms suggestive of liver dysfunction appear. ${ }^{4,8}$ Patients should be assessed by a health care professional; if hepatotoxicity is ruled out, isoniazid can be restarted. Given the low bacillary burden of latent tuberculosis infection and the prolonged need for therapy, it is unlikely that a brief interruption of treatment would affect outcome.

\section{Role of the medical team}

Patients, their families, pharmacists, the prescribing physicians, public health nurses, and physicians involved in follow-up care all have key roles in preventing adverse events related to isoniazid treatment. Patients and their families need to be aware of the signs and symptoms of hepatotoxicity and the actions to take in the event of their occurrence.

Since this patient's case, our tuberculosis clinic has developed a standard form to be given to all patients at the start of treatment that details adverse effects and the actions to take if they develop (Appendix 1, available at www.cmaj.ca /lookup/suppl/doi:10.1503/cmaj.121732/-/DC1). This guide is consistent with the American Thoracic Society and CDC guideline, ${ }^{3}$ which recommends that printed instructions including clinic telephone numbers and explicit instructions for after-hours care be given to patients. If symptoms develop, patients are told to stop taking the medication immediately and contact a health care provider. The health care provider can then triage the urgency of evaluation, with the default plan being to see the patient in the emergency department for assessment.

Patients at our clinic are still followed monthly and receive reminders about symptoms to monitor at each visit. We do not routinely monitor liver function, doing so only if symptoms develop or any of the recommended criteria for monitoring are met. ${ }^{4,8}$ Our clinic collaborates closely with public health tuberculosis nurses and staff involved in directly observed therapy, who monitor active cases daily and children receiving treatment for latent tuberculosis infection every 2 weeks. When English is a patient's second language, a translator or telephone translator service is used to communicate this information.

\section{References}

1. Kitai I, Malloy P. A clinical approach to paediatric tuberculosis in Canada. Paediatr Child Health 2003;8:162-70.

2. Centers for Disease Control and Prevention (CDC). Severe isoniazid-associated liver injuries among persons being treated for latent tuberculosis infection — United States, 2004-2008. MMWR Morb Mortal Wkly Rep 2010;59:224-9.

3. Targeted tuberculin testing and treatment of latent tuberculosis infection. This official statement of the American Thoracic Society was adopted by the ATS Board of Directors, July 1999. This is a Joint Statement of the American Thoracic Society (ATS) and the Centers for Disease Control and Prevention (CDC). This statement was endorsed by the Council of the Infectious Diseases Society of America. (IDSA), September 1999, and the sections of this statement. Am J Respir Crit Care Med 2000;161:S221-47.

4. Ellis E, Long R, Elwood K. Canadian tuberculosis standards. 6th ed. Ottawa (ON): Public Health Agency of Canada; 2007.

5. Lee WM. Drug-induced hepatotoxicity. N Engl J Med 1995;333: 1118-27.

6. Nolan CM, Goldberg SV, Buskin SE. Hepatotoxicity associated with isoniazid preventive therapy: a 7-year survey from a public health tuberculosis clinic. JAMA 1999;281:1014-8.

7. Salpeter SR. Fatal isoniazid-induced hepatitis. Its risk during chemoprophylaxis. West J Med 1993;159:560-4.

8. Saukkonen JJ, Cohn DL, Jasmer RM, et al. An official ATS statement: hepatotoxicity of antituberculosis therapy. Am J Respir Crit Care Med 2006;174:935-52.

9. Devrim I, Olukman O, Can D, et al. Risk factors for isoniazid hepatotoxicity in children with latent TB and TB: difference from adults. Chest 2010;137:737-8.

10. Wu SS, Chao CS, Vargas JH, et al. Isoniazid-related hepatic failure in children: a survey of liver transplantation centers. Transplantation 2007;84:173-9.

11. Moulding TS, Redeker AG, Kanel GC. Twenty isoniazidassociated deaths in one state. Am Rev Respir Dis 1989;140:700-5.

Affiliations: From the Division of Infectious Diseases (Science, Kitai), The Hospital for Sick Children, Toronto, Ont.; the Department of Clinical Epidemiology and Biostatistics (Science), McMaster University, Hamilton, Ont.; the Division of Clinical Pharmacology and Toxicology (Ito), The Hospital for Sick Children, Toronto, Ont.; and the Department of Paediatrics (Ito, Kitai), University of Toronto, Toronto, Ont.

Contributors: Ian Kitai conceived the idea for the report. All of the authors were involved in drafting the article, revising it critically for important intellectual content and approving the final version submitted for publication.

Acknowledgements: The authors thank Elizabeth Rea, who provided invaluable feedback on the manuscript. 